Revue d'histoire de l'Amérique française

REYUE D.HISTOIRE DE L'AMÉRIQUE FRANÇAISE

\title{
Les engagements pour la traite des fourrures
}

Évaluation de la documentation

\section{Gratien Allaire}

Volume 34, numéro 1, juin 1980

URI : https://id.erudit.org/iderudit/303835ar

DOI : https://doi.org/10.7202/303835ar

Aller au sommaire du numéro

Éditeur(s)

Institut d'histoire de l'Amérique française

ISSN

0035-2357 (imprimé)

1492-1383 (numérique)

Découvrir la revue

Citer cet article

Allaire, G. (1980). Les engagements pour la traite des fourrures : évaluation de la documentation. Revue d'histoire de l'Amérique française, 34(1), 3-26.

https://doi.org/10.7202/303835ar d'utilisation que vous pouvez consulter en ligne.

https://apropos.erudit.org/fr/usagers/politique-dutilisation/ 


\title{
LES ENGAGEMENTS POUR LA TRAITE DES FOURRURES
}

\author{
évaluation de la documentation* \\ GRATIEN ALLAIRE \\ Faculté Saint-Jean \\ The University of Alberta
}

Plusieurs historiens ont cru, comme Louise Dechêne, que l'habitude était prise dès le début du XVIIIe siècle «de consigner toutes les transactions relatives à la traite dans des contrats notariés". Par conséquent, selon elle, «les répertoires des notaires peuvent être considérés comme de véritables registres d'enregistrement des allées et venues dans l'Ouest "' ${ }^{\prime}$. Se basant sur cette documentation, l'historienne trace un portrait très détaillé du personnel de la traite pour la décennie 1708-1716, portrait dont la principale caractéristique est la concentration, régionale et familiale. Elle y distingue les «voyageurs indépendants" des "engagés pour la traite», ces derniers étant au service des premiers ${ }^{2}$.

Dans un article récent, Fernand Ouellet a sérieusement remis en question l'utilisation des archives notariales comme source documentaire principale: «L'historien, écrit-il, qui désire évaluer sur une base annuelle le volume de la main-d'oeuvre engagée dans le commerce des pelleteries ne saurait confiner son enquête au Répertoire des engagements pour l'ouest et aux actes notariés. ${ }^{3}$ La documentation de ce dernier, les Trade Licences et la collection Ermatinger, porte surtout sur l'après-Conquête. Cependant, sa critique porte aussi sur les années précédentes:

* Communication présentée au congrès annuel de l'Institut d'histoire de l'Amérique française, le 19 octobre 1979.

1 Louise Dechêne, Hahitants et marchands de Montréal au XVIle siècle (Montréal, Plon, c1974), 217. Aussi: Marcel Trudel, Initiation à la Nouvelle-France. Histoire et institutions (Montréal, Holt, Rinehart et Winston, c1968), 210; W.J. Eccles, The Canadian Frontier, 1534-1760 (Toronto, Holt, Rinehart and Winston, c1969), 146; W.J. Eccles, France in America (Toronto, Fitzhenry \& Whiteside, c1972), 146. Cette dernière description de Eccles serait plus juste si elle était appliquée à la Compagnie du Nord-Ouest.

2 Dechêne, Habitants et marchands, 217-229

3 Fernand Ouellet, "Dualité économique et changement technologique au Québec (1760-1790)", Histoire Sociale / Social Historl, IX, 18 (nov. 1976): 294-295.

RHAF, vol. 34, n 1 , juin 1980 
Également intéressant à cet égard est le congé de traite accordé à La Vérendrye en 1747 pour quatre canots ayant chacun six hommes d'équipage, soit 24 hommes. Le congé indique les noms de 14 engagés du sieur Gonneville alors que le Répertoire ne fait état que de trois engagements de la part de Gonneville et de onze par La Vérendrye. ${ }^{4}$

Une telle critique vise la base même du portrait tracé par Louise Dechêne: étant donné que, sur ce sujet, elle n'a utilisé que les actes notariés, sa documentation risque d'être trop restreinte pour que son tableau soit valable.

La présente communication cherche à faire le point sur le sujet par une critique plus poussée des sources. L'analyse porte principalement sur les contrats d'engagement pour la traite des fourrures retrouvés dans les archives notariales pour les années 1701 à 1745 . Bien qu'ils ne représentent qu'une partie de la masse documentaire relative au commerce des fourrures, ces contrats peuvent être considérés comme une large fenêtre ouverte sur ce secteur économique. Cependant, leur nombre et leur valeur documentaire varient selon les périodes. La communication a pour but non seulement de décrire la provenance de la documentation et d'en établir les caractéristiques chronologiques, mais encore d'en évaluer la représentativité au moyen d'autres documents notariés et des congés.

\section{ORIGINE DE LA DOCUMENTATION}

Edmond-Zotique Massicotte a déjà dressé un répertoire des contrats d'engagement passés devant notaire, rendant ainsi plus accessible une documentation importante que les historiens ont peu ou pas exploitée ${ }^{5}$. Toutefois, cette liste n'est pas très fiable. D'une part, elle ne contient pas tous les contrats d'engagement passés devant notaire. En effet, certains contrats furent omis; de plus, dans le cas de contrats collectifs, la liste des engagés n'est pas toujours complète; enfin, seul le dépôt de Montréal a été dépouillé. D’autre part, Massicotte y a répertorié d'autres contrats relatifs à la traite: des marchés-permissions de traite pour le Détroit entre 1706 et 1716 et des «obligations» envers la Compagnie des Indes occidentales contenues au minutier du notaire Claude-Cyprien-Jacques Porlier. Et

4 Ibid.: 295, aussi 260.

5 E.-Z. Massicotte, "Répertoire des engagements pour l'Ouest conservés dans les Archives judiciaires de Montréal", RAPQ (1929-1930): 191-466. Cette première tranche porte sur les engagements précédant 1745 . 
ces inclusions ne sont pas systématiques. Par conséquent, ce répertoire ne peut servir que de point de départ.

Tous les minutiers subsistants des notaires ayant exercé entre 1701 et $1745^{6}$ ont donc été dépouillés et seuls ont été retenus les engagements pour la traite des fourrures. Les contrats pour le service du roi de même que les contrats annulés ont été écartés. Le nombre des engagements retrouvés s'élève à 5964 . Le tableau I en donne la

\section{TABLEAU I}

\section{Notaires et engagements, 1701-1745}

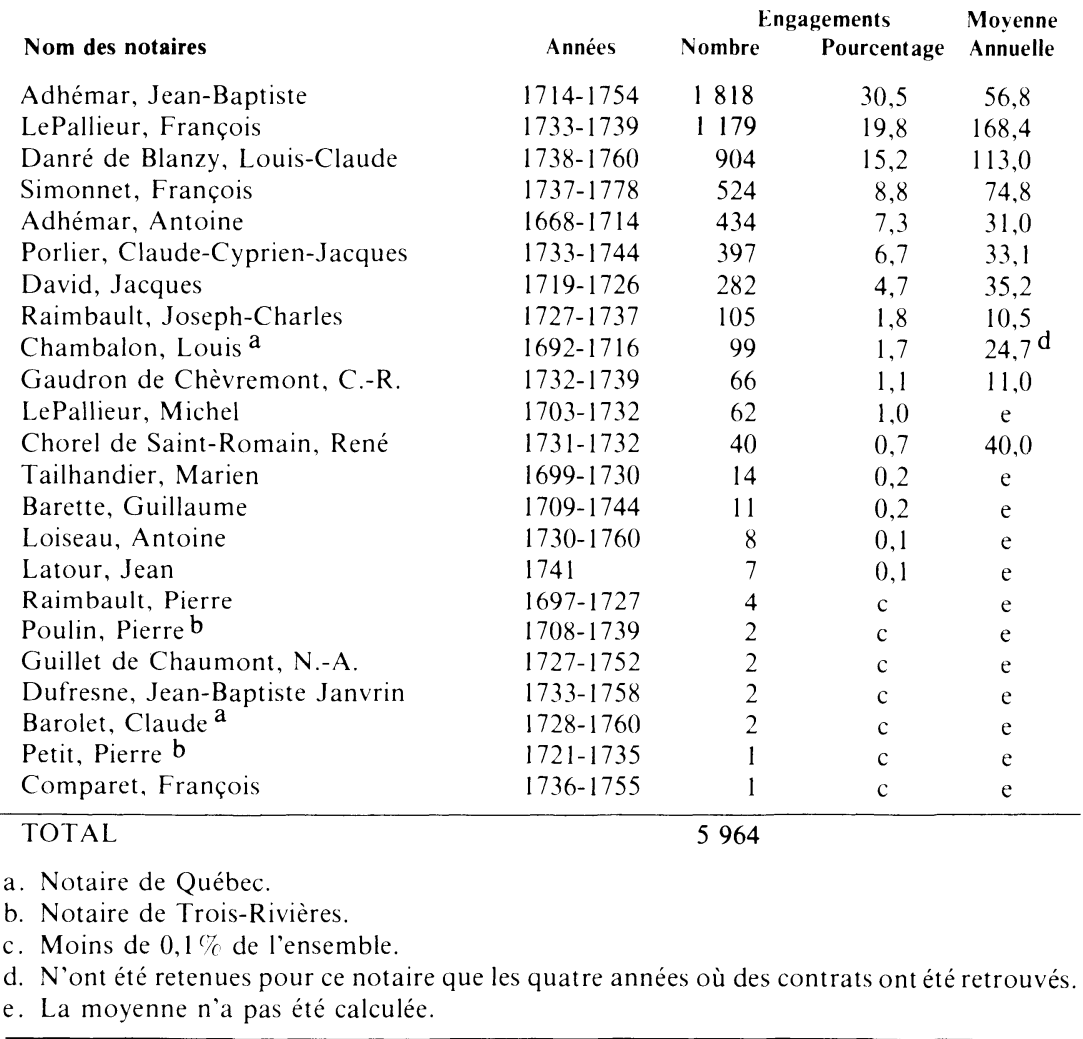

6 Peter N. Moogk a évalué à un sixième les documents notariés perdus, sans toutefois différencier les gouvernements. "Manual Education and Economic Life in New France", Travaux sur Voltaire et le dix-huitième siècle, no 167 (1977): 127-128. 
répartition. La très grande majorité se trouve dans les archives notariales de Montréal: 5860 engagements, 98,2\%. Les recherches dans les archives de Québec ont permis d'en repérer 101. Le notaire Louis Chambalon a rédigé la plupart de ces derniers entre 1702 et 1705 pour le compte de la Compagnie de la colonie, dont le siège social était à Québec ${ }^{7}$. Le dépouillement des archives notariales de TroisRivières n'a rapporté que trois contrats ${ }^{8}$. La récolte aurait dû être meilleure puisque cette ville était un "nid d'éclosion des "voyageurs' ${ }^{9}$ et un centre de fabrication de canots d'écorce, et que les noms de lieux de la région reviennent souvent dans les contrats passés à Montréal.

Les notaires exerçant "dans les côtes" du gouvernement de Montréal ont rédigé très peu de contrats d'engagement. Certains, comme François Coron et Jean-Baptiste Tétro de la Pointe-auxTrembles, n'en ont passé aucun. D'autres n'en reçurent que quelques-uns: Guillaume Barette et Antoine Loiseau, de la rive sud, en rédigèrent à peine 19 sur une période de 35 ans. La rédaction des contrats est donc fortement concentrée à Montréal même, où elle se faisait généralement à l'étude du notaire. Bien plus, un très petit nombre de notaires se sont partagé la plus grande partie du travail. Deux notaires, Jean-Baptiste Adhémar et François LePallieur, ont rédigé, ensemble, $50,3 \%$ des engagements retrouvés. Deux autres, Louis-Claude Danré de Blanzy et François Simonnet, se partagent $24 \%$ de la "population" observée. En fait, $96,3 \%$ des engagements se trouvent dans le minutier de 9 des 43 notaires ayant exercé dans la juridiction de Montréal durant ces 45 ans.

La répartition selon les moyennes annuelles est un moyen plus sûr de connaître l'importance d'un notaire. Selon ce critère, François LePallieur occupe le premier rang. Il n'a exercé que 7 ans et recueilli 1179 contrats, dont seulement 30 durant la première et la dernière année de sa pratique. Ces deux années exclues, il rédigea en

ANQQ, L. Chambalon, 17 avril 1702, no 2198; 29 mars 1702, no 2269; 15 mai 1702, no 2302 ; $14-27$ juin 1702 , no 2310 ; 14 juin 1702 , no $2311 ; 24$ juin 1702 , no 2319 ; 25 juin 1702 , no 2321 ; $14-15$ sept. 1702 , no 2359 ; 2 juin 1704 , no $2932 ; 25$ juin 1704 , no $2938 ; 27$ juin 1704 , no $2965 ; 2$ avril-14 mai 1705 , no $3163 ; 8$ avril-19 mai 1705 , no $3166 ; 21-28$ avril 1705 , no 3174 ; 29 juil. 1707, no $3562 ; 8$ mai 1713 , no $4494 ; 12$ sept. 1713, no 4548. Les autres contrats ont été retrouvés dans le minutier de Claude Barolet: 5 mai 1741, no 1010;16 mai 1741, no 1012. Engagements à Pierre Gaultier de La Vérendrye.

8 ANQTR, P. Poulin, 20 avril 1716, no 70; 7 mars 1721, no 206; P. Petit, 19 fév. 1733, no 115 .

9 Benjamin Sulte, Histoire des Canadiens-français, 1608-1880. Tome VI (Montréal, Wilson et Cie, 1882), ( Montréal Éditions Élysée, 1977), 138. 
moyenne 229,8 contrats par année. Viennent ensuite L.-C. Danré de Blanzy et F. Simonnet. Ils exercèrent à partir de 1737 et, avant 1746 , ils recueillirent respectivement 113,0 et 74,8 engagements par année. J.-B. Adhémar, qui en a rédigé le plus grand nombre, vient ensuite. Il reçut en moyenne 56,8 engagements par an durant ses 32 années de pratique. Quant aux autres notaires, ils sont de moindre importance. Cette concentration signifie une grande uniformité dans la rédaction des contrats. C'est particulièrement le cas de F. LePallieur, Danré de Blanzy et Simonnet dont la pratique est de courte durée et le nombre de contrats élevé. De fait, l'un et l'autre utilisent une formule standardisée, écrite au préalable par un clerc, de sorte qu'il n'a plus qu'à remplir les blancs.

\section{ÉVOLUTION DE LA TRAITE ET ENGAGEMENTS}

L'origine de la documentation 'iant établie, il s'agit ensuite de voir sa répartition dans le temps. L'année utilisée pour les calculs représente l'année de départ de l'engagé pour les pays d'en haut. Ainsi, les contrats passés en novembre et décembre d'une année sont reportés à l'année suivante. Le tableau II présente la répartition annuelle des engagements. À sa lecture, une première constatation s'impose: il y a un énorme contraste entre les trente premières et les quinze dernières années. Celles-ci totalisent 4168 engagements, soit $69,9 \%$ de l'ensemble. Avant 1730, 4 années seulement dépassent les 100 engagements; à partir de 1731 , seules les trois premières n'atteignent pas 250 . La moyenne annuelle des 30 premières années s'élève à 59,9 engagements; celle des quinze dernières se situe à 277,9 soit quatre fois et demie plus élevée.

Dans l'ensemble, l'histoire du commerce des fourrures fournit les jalons nécessaires à une meilleure compréhension de la situation et de son évolution. Une date-charnière s'offre dès l'abord: l'année 1716 , année du rétablissement par le roi du système de congés. Il s'agit là d'un point tournant. La première moitié du XVIIle siècle est ainsi divisée en deux époques. La première, de 15 ans, est caractérisée par la confusion et les problèmes; elle marque, pour employer l'expression de Guy Frégault, "l'effondrement du commerce du castor $"{ }^{10}$. La seconde, longue de trente ans, est plus prospère: les prix sont plus stables, le commerce est réorganisé à la faveur d'une nouvelle politique amérindienne et connaît, en deux étapes, une expan-

\footnotetext{
10 Guy Frégault, «La Compagnie de la colonie», Le XVIIIe siècle canadien. Études (Montréal, Éditions HMH, 1968), 280.
} 
8

REVUE D'HISTOIRE DE L'AMERIQUE FRANÇAISE

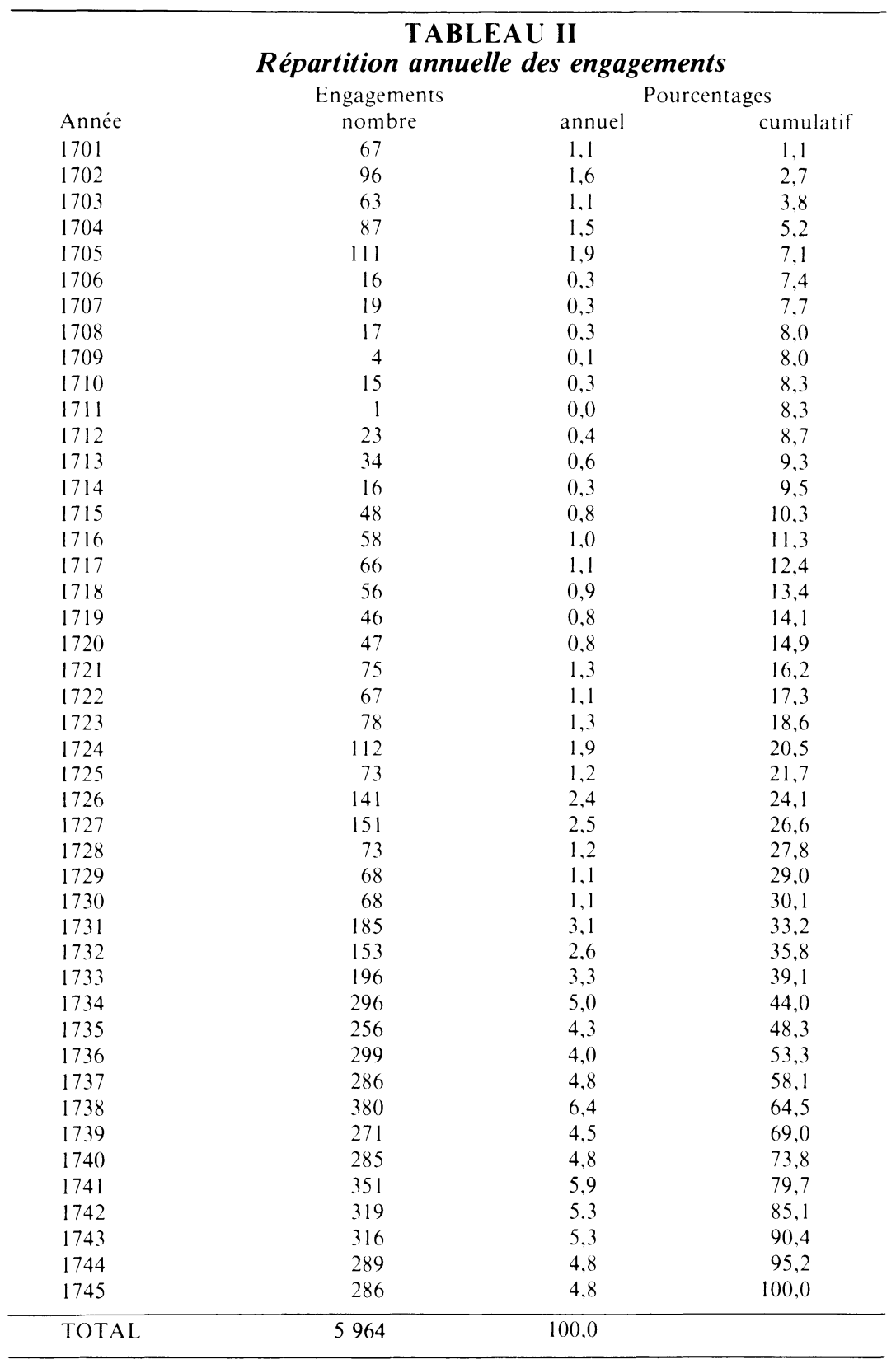


sion sans précédent. Les engagements de la première période seront confrontés à d'autres documents notariés; ceux de la seconde seront examinés à l'aide des renseignements fournis par les congés de traite.

\section{PREMIẼRE ÉPOQUE: 1701-1715}

Le XVIIIe siècle s'est ouvert sur une crise, causée par un surplus de castors, surplus que ne peut absorber le marché métropolitain. Le problème n'était pas nouveau et avait déjà amené les autorités métropolitaines à prendre des mesures draconiennes: abolition, en 1696, du système de congés établi en 1681 dans le but de régulariser l'approvisionnement; abandon des postes de l'ouest, à quelques exceptions près. Ces mesures devaient, selon les autorités métropolitaines, produire une réduction appréciable de la production annuelle de castor et avoir pour conséquence de forcer la consommation des peaux de castor en inventaire dans les entrepôts de France et de la colonie.

Ces décisions de la métropole plaçaient la colonie dans une situation difficile tant au point de vue économique qu'au point de vue militaire et politique. La bourgeoisie de la fourrure ", avec l'approbation royale, décida de mettre sur pied sa propre organisation. La Compagnie de la colonie contrôlait et l'achat des fourrures dans la colonie, l'approvisionnement, et leur vente en France, la mise en marché. Elle dut faire face à de nombreux problèmes qu'elle ne put résoudre ${ }^{12}$. Ruinée, elle conclut, au début de 1706, une entente avec Louis-François Aubert, Jean-Baptiste Néret et Jean-Joseph Gayot. Ces derniers se chargeaient de la mise en marché des castors en Europe.

L'entente produisit un effet immédiat dans la colonie. Comme le prix fixé pour le castor sec était bas, que le castor gras n'était pas accepté par les associés et que les fourrures en général se vendaient à meilleur prix à Albany, la contrebande s'installa dans la colonie, avec la quasi-tolérance des autorités coloniales ${ }^{13}$. À cause de la désorganisation du commerce, les nations amérindiennes alliées des Français se rapprochèrent des marchands de fourrures d'Albany et de New York. Comme remède à cette situation dangereuse pour le

11 Pour utiliser l'expression de W.L. Morton. The Kingdom of Canada, A General History from Earliest Times (Toronto, McClelland and Stewart Limited, c1973), 54.

12 Frégault, "La Compagnie de la colonie», 263-269.

13 Ibid., 279. 
commerce et la sécurité de la Nouvelle-France, les autorités coloniales, Vaudreuil en tête, réclamèrent le rétablissement des congés.

Les arguments de la colonie n'avaient pas suffisamment de poids pour amener le ministre de la Marine à modifier sa politique. Ce n'est que lorsque les surplus de castor entreposés depuis longtemps à Québec eurent été éliminés sous l'effet combiné de la pourriture et de la vermine ${ }^{14}$ que le ministre Pontchartrain consentit, à titre d'essai seulement, à l'émission de 15 congés. L'annonce en fut faite aux autorités coloniales par le mémoire du roi du 10 juillet $1715^{15}$. Les congés ne furent pas émis cette année-là ${ }^{16}$. Ils le furent plutôt en avril $1716^{17}$. Le nombre plus élevé d'engagements en 1715 semble avoir été le résultat des permis spéciaux émis en échange de la participation de leur détenteur à la guerre des Renards ${ }^{18}$.

Après la mort de Louis XIV, et à la suite de la formation d'un Conseil de la Marine, Vaudreuil réussit à faire accepter son point de vue au sujet du commerce des fourrures et de la diplomatie amérindienne. Les congés furent alors rétablis officiellement et leur nombre porté à 25 . La déclaration du roi est datée du 28 avril 1716; elle fut enregistrée au Conseil supérieur le ler décembre suivant ${ }^{19}$. Ce n'est fort probablement qu'en 1717 que recommença l'émission annuelle de 25 congés. En effet, malgré une disposition à cet effet ${ }^{20}$, aucun congé ne paraît avoir été enregistré à Montréal avant 1717 . En plus des congés rétablis, le gouverneur pouvait en vertu de l'amnistie accordée en mars 1716 aux coureurs de bois, émettre dés permissions spéciales de "faire la traite afin de (les) indemniser des frais du Voyage" à ceux qui acceptaient de prendre part aux guerres

14 Eccles, France in America, 119.

15 Yves F. Zoltvany, Philippe de Rigaud de Vaudreuil: Governor of New France, 1703-1725 (Toronto, McClelland and Stewart, c1974), 144.

16 Yves F. Zoltvany, Philippe de Rigaud de Vaudreuil: Governor of New France, (1703-1725) (Edmonton, The University of Alberta, 1963), 247.

17 Zoltvany, Vaudreuil (c1974), 154, note 64, "Congés, avril 1716..." Autre confirmation dans une ordonnance de l'intendant Michel Bégon du 10 avril 1716: Pierre-Georges Roy, Inventaire des ordonnances des intendants de la Nouvelle-France conservées aux Archives provinciales de Québec (Beauceville, L'Éclaireur, 1919), I: 161.

18 Zoltvany, Vaudreuil (c1974), 141.

19 Jugements et délibérations du Conseil Supérieur de Québec, vol. VI, 7 janvier 1710-22 décembre 1716 ( Québec, Imprimerie Joseph Dussault, 1891), 1213. Le texte de cette déclaration fut enregistré à Montréal, à la suite de l'amnistie accordée aux coureurs de bois : ANQM, Registre des audiences de la juridiction royale de Montréal, 8 (1714-1719): ff. 1249$1250 \mathrm{v}$.

20 ANQM, Registre des audiences, 8: f. 1250 
amérindiennes ${ }^{21}$. Commençait alors une nouvelle phase du commerce des fourrures 22 .

Cette époque d'une durée de quinze ans, qui précède le rétablissement des congés, compte 617 engagements, soit 10,3\% de l'ensemble. La moyenne annuelle s'élève à 41,1 engagements. L'époque peut être divisée en deux périodes bien distinctes: celle de la Compagnie de la colonie, de 1701 à 1705 , et la période de "confusion", de 1706 à 1715. Elles contrastent fortement, comme le laisse voir le graphi-

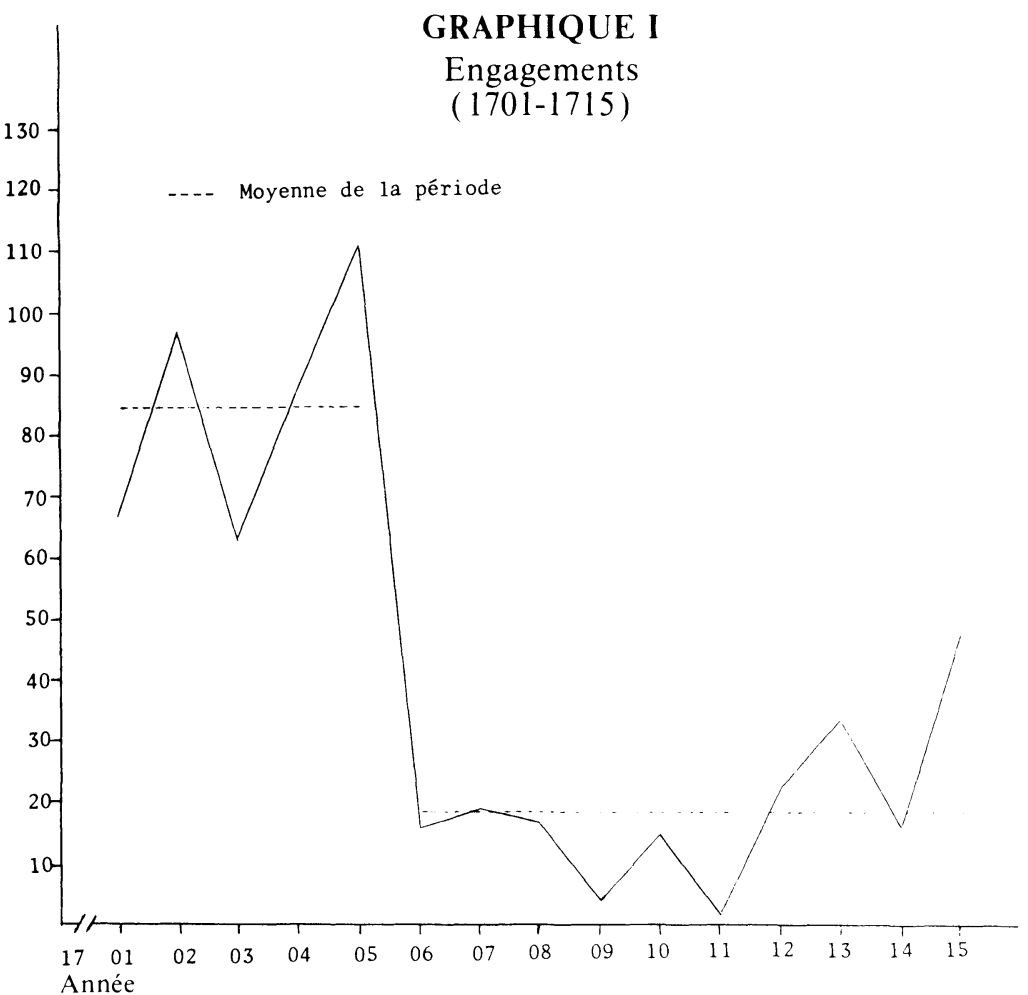

$21 \quad$ Ibid.: ff. $1245 \mathrm{v}-1247$, art. 5.

22 La description la plus claire des étapes du rétablissement des congés se trouve dans Zoltvany, Vaudreuil (c1974), 143-151. Harold A. Innis ( The Fur Trade in Canada. An Introduction to Canadian Economic History (Toronto, University of Toronto Press, c 1970, 106) date le rétablissement de 1715: Emile Salone (La colonisation de la Nouvelle-France. Étude sur les origines de la nation canadienne-française (Paris, Guilmoto, s.d.); ( Trois-Rivières, Le Boréal Express, 1970, 392-393) et Alice Jean Élizabeth Lunn, Economic Development in New France, 1713-1760 (Montreal, McGill University, 1942, 120) le font dater de 1716. 
que I. Chacune des cinq années de la première dépasse la moyenne de l'époque; une seule des dix années de la seconde le fait. La première totalise 424 engagements, $68,7 \%$ du total de l'époque; la seconde n'en regroupe que 193 , soit $31,3 \%$. La moyenne annuelle de la première s'élève à 84,8 engagements; celle de la seconde à 19,3.

Le repérage des contrats de la première période s'est avéré plutôt facile. Contrats collectifs, ils avaient été rédigés par Antoine Adhémar à Montréal ou Louis Chambalon à Québec: $75 \%$ et 22,6\% des engagements respectivement se trouvent dans leur minutier. Une concentration à peu près égale se retrouve dans la seconde période. Antoine Adhémar a, durant ces années encore, reçu le plus d'engagements: 116 des 193 , soit $60,1 \%$. Si l'on ajoute à ces derniers ceux qu'a reçus le fils d'Antoine, Jean-Baptiste ${ }^{23}$, le total s'élève alors à 162 et le pourcentage à 83,9. Un troisième notaire, Michel LePallieur, n'a rédigé que $10,9 \%$ des engagements, en dépit du fait qu'il habitait Montréal même et qu'il exerça sa profession durant toute la période.

Le repérage des contrats de cette seconde période s'est révélé une entreprise difficile et hasardeuse. Dans le minutier d'Antoine Adhémar, le titre inscrit sur le contrat est souvent trompeur: en effet, le notaire a fréquemment confondu engagement et marchépermis de traite. L'engagement consiste en la location de sa force de travail en échange d'une rémunération fixe, indiquée au contrat. Le marché-permis de traite ne comporte pas de rémunération fixée d'avance. Il s'agit plutôt d'une entente entre un ou plusieurs voyageurs et le commandant d'un poste, Détroit et Antoine de Lamothe Cadillac en l'occurrence, selon laquelle le voyageur, ou les voyageurs en société, acceptent de transporter des marchandises à l'aller et des pelleteries au retour, en échange d'un permis de faire la traite au poste en question.

Ces marchés-permis n'ont pas tous été relevés. Cependant, l'analyse de deux d'entre eux aide à mieux connaître l'utilisation de l'engagement durant cette période. Le premier date d'avril 1707; le notaire l'a intitulé "Mr. de Lamothe Cadillac, plusieurs engagements pour le Détroit» ${ }^{24}$. Les détenteurs de ces permis devaient transporter, dans chacun des sept canots mentionnés au document, 300 livres pesant de marchandises à l'aller et le même poids de pelle-

23 Il remplaça son père à la mort de celui-ci.

24 ANQM, A. Adhémar, 2-11 avril 1707, no 7668. 
teries au retour, pour le compte de Lamothe Cadillac. Ce service était gratuit «En Considera'on de Ce quil (Lamothe) Leur permet de traicter Les marchandises qls porteront pour Eux estans arrivez aud fort Le pontchartrain» 25 . Fait plus important encore, sur les 24 hommes se liant ainsi à l'agent de Lamothe Cadillac, un seul est identifié comme engagé. Le deuxième contrat, similaire, a été passé en septembre de la même année. Dans ce cas, un des trois hommes est à l'emploi des deux autres ${ }^{26}$. Ces deux documents suggèrent le peu d'importance de l'engagement durant cette période.

L'étude d'une partie du minutier de Michel LePallieur donne du poids à cette hypothèse. Ce notaire a rédigé peu de contrats d'engagement. Il a cependant reçu plusieurs autres contrats relatifs à la traite. Un relevé sommaire de ceux de 1713 donne les résultats suivants: 90 «obligations ${ }^{27}$ et 3 contrats de société, en plus de 5 contrats d'engagement. Aux 5 engagés, les autres contrats opposent 115 personnes différentes ${ }^{28}$. Cette même année 1713, Antoine Adhémar rédigeait 27 engagements. Quoique l'inventaire des minutes de ce dernier ait été publié, il est impossible de faire les mêmes calculs. En effet, les titres à l'inventaire sont trop vagues ${ }^{29}$. De plus, aucun inventaire n'a été publié du greffe de Pierre Raimbault, autre notaire de Montréal. Il n'a reçu aucun engagement entre 1706 et 1715, et 4 seulement entre 1716 et 1727 . À partir de 1718 cependant, il rédigea de nombreuses reconnaissances de dettes relatives à la traite ${ }^{30}$. Il apparaît donc que l'engagement est très peu utilisé durant cette période pour le recrutement du personnel de traite.

Toutes ces indications sont encore confirmées par les recherches de Louise Dechêne ${ }^{31}$. La période qu'elle étudie est à peine différente: 1708-1717 au lieu de 1706-1715. Elle a consulté tous les documents notariés pour la période. Ils contiennent 1120 départs pour l'ouest; de ce nombre, 267 seulement, ou $23,8 \%$, étaient des engagés ${ }^{32}$. Le tableau II en indique 282 pour les mêmes années, soit $25,2 \%$ des départs recensés par Dechêne. D'après le graphique tracé

25 Ibid., 4 avril 1707

26 ANQM, A. Adhémar, 22 sept. 1707, no 7820. Ce contrat est répertorié comme engagement par Massicotte ( Répertoire», 211).

27 Reconnaissance de dette.

28 ANQM, M. LePallieur, année 1713.

29 Antoine Roy, Inventaire des Greffes des Notaires du Régime français, VI (Antoine Adhémar) (Québec, 1945): 287-308.

30 Massicotte, "Répertoire", 223ss.

31 Dechêne, Hahitants et marchands, 217-229.

32 Ihid., 219. 
par cet auteur ${ }^{33}$, la différence entre les données se retrouve durant les années 1713 à 1717. Il s'agit fort probablement d'une interprétation différente de certains documents. Un fait demeure: les engagés sont loin d'être la majorité du personnel de la traite. Le graphique de Dechêne montre toutefois que le fossé entre le nombre de voyageurs indépendants et le nombre d'engagés se comble considérablement à partir de 1715, année de la participation à la guerre des Renards, suivie du rétablissement des congés.

Ce faisceau d'indices tirés des contrats notariés tend vers une conclusion: durant cette période, l'engagement n'est pas la formule privilégiée de participation à la traite des fourrures. Bien sûr, les contrats relatifs à la traite n'ont pas tous été passés devant un notaire. Même, il est fort probable que les engagements aient été souvent faits par entente verbale ou sous seing privé ${ }^{34}$, certainement plus souvent que les contrats intéressant directement un marchand-équipeur. Toutefois, l'écart noté entre engagés et "partenaires» est tellement grand qu'il faudrait que le phénomène soit très prononcé pour infirmer cette conclusion.

Il ressort nettement des données analysées que le type d'organisation décrit par Lahontan dans ses Voyages ${ }^{35}$ domine la traite. L'association, soit au moyen d'un contrat de société, soit par la signature d'une "obligation solidaire" ${ }^{36}$, représente la forme de participation la plus utilisée entre 1706 et 1715 . Elle n'est praticable, cependant, qu'aux moments ou aux endroits où l'investissement initial reste relativement peu élevé et où la réalisation des bénéfices se fait en un laps de temps relativement court ${ }^{37}$. De plus, l'illégalité

33 Ibid., 512.

34 De tels contrats sont parfois mentionnés dans d'autres contrats. Par exemple, Jean et Dominique Garo dans une "obligation" de Pierre Garo dit St-Onge à François Ardouin, ANQM, A. Adhémar, 31 juillet 1708, no 8082; Jean-Baptiste Jarry et Noël Lefebvre dit Lasiseray dans une "obligation" de Pierre Magdelaine dit Ladouceur à Pierre de Lestage, ANQM, P. Raimbault, 26 mai 1720, no 2599; Jacques Vaudry dans une obligation de Jean Charpentier à Jean-Baptiste Neveu, ANQM, M. LePallieur, 25 avril 1707, no 458. Aussi: ANQM, Registre des audiences de la juridiction rolale de Montréal, 8( 1714-1719): ff. 1307v1308, où il est fait mention, pour le même voyage, d'un engagement sous seing privé et d'un autre devant J.-B. Adhémar.

35 Baron de Lahontan, Voyages du Baron de la Hontan dans l'Amérique septentrionale..., Tome I (Amsterdam, MDCCV); ( Montréal, Éditions Élysée, 1974), 79-81. Une description similaire du même auteur est reprise dans: Normand Lafleur, La vie traditionnelle du coureur de bois au XIXe et XXe siècle (Montréal, Leméac, 1973), 50-51.

36 Reconnaissance de dette selon laquelle les débiteurs se déclarent tous responsables de la dette contractée.

37 Voir à ce sujet: Innis, The Fur Trade, 110-111. 
plus ou moins réelle de la traite favorise cette répartition des risques. Avec le changement graduel de ces conditions se produit également une modification de l'organisation de la traite.

\section{DEUXIÈME ÉPOQUE: 1716-1745}

Le rétablissement des congés ouvre une seconde période du commerce des fourrures. Il constitue un changement d'importance des conditions d'approvisionnement et coïncide avec le début d'une période de plus grande stabilité de ce secteur. Les surplus de castor ayant été écoulés ou détruits, le marché français absorbe plus rapidement la production coloniale. En 1717, la Compagnie de l'Occident, suivie de la Compagnie des Indes, prend en charge le monopole du castor et en assure la mise en marché. Par la suite, estime L. Dechêne, "les variations de prix sont fréquentes mais peu prononcées " ${ }^{38}$. A cette nette amélioration du secteur du castor s'ajoute un accroissement de la demande de menues pelleteries et de peaux. Comme les autorités métropolitaines modifient au même moment leur politique relative à la traite et à la diplomatie amérindienne, les conditions d'un nouvel essor se trouvent réunies ${ }^{39}$.

Ces changements dans la métropole se répercutent sur la colonie, lieu de l'approvisionnement. Ils produisent en premier lieu une expansion en deux étapes du territoire de traite. Avant 1730, les Français réoccupent les rives des Grands lacs, régions exploitées au XVIIe siècle. Après 1730, à la suite de Pierre Gaultier de Varennes de La Vérendrye, ils s'engagent à l'ouest du lac Supérieur ${ }^{40}$. Reliées à cette expansion, les guerres amérindiennes, en particulier contre les Renards, exercent, durant ces trente années, une influence indiscutable sur le commerce ${ }^{41}$. Enfin, autre résultat de l'expansion, la

38 Dechêne, Habitants et marchands, 146.

39 Ceci ne veut pas dire que tous les problèmes sont réglés. En effet, la contrebande avec la colonie de New York continue, particulièrement entre 1735 et 1738. Maurice Filion, La pensée et l'action coloniales de Maurepas vis-à-vis du Canada, 1723-1749. L'âge d'or de la colonie (Montréal, Leméac, 1972), 359-364.

40 A.J.E. Lunn a dressé la liste de ces postes et de leur date d'établissement, (Economic Development, 450-452). Les explorations de La Vérendrye et ses difficultés sont décrites dans les ouvrages d'Antoine Champagne, Les La Vérendrye et le poste de l'Ouest (Québec, Les Presses de l'Université Laval, 1968), x-592 p.; Nouvelles études sur les La Vérendrye et le poste de l'Ouest (Québec, Les Presses de l'Université Laval, 1971), 200 p.

41 Les guerres a mérindiennes dans ce secteur n'ont pas encore fait l'objet d'une étude poussée. Certains renseignements peuvent être tirés des études d'Eccles (The Canadian Frontier, 148-149), d'Innis (The Fur Trade, 90-91), de Gustave Lanctôt (Histoire du Canada, vol. III, Du Traité d'Utrecht au Traité de Paris, 1713-1763 (Montréal, Beauchemin, 1964): 5355 ) et, plus récemment, de Zoltvany (Vaudreuil ( c1974), 112-123, 127, 134-136, 140-143, 165 166, 193-194 et 208). 
formule d'exploitation des territoires de traite subit des transformations profondes.

Durant ces trente années, la traite des fourrures à l’intérieur du continent prend des formes nouvelles, variant selon le bon plaisir et l'imagination du ministre de la Marine et/ou du gouverneur de la colonie. Ce dernier apprend, dès 1718 , que le système de congés, à peine rétabli, serait de nouveau aboli à partir de 1720 et qu'aucun autre permis ne pourrait être émis ${ }^{42}$. Divers délais, pour diverses raisons, reportent la fin du système à l'année $1723^{43}$. Devant la concurrence des marchands anglais, les autorités métropolitaines décident un peu plus tard de faire revivre, à peine modifié, le système de congés. Cette décision, le roi la communique au gouverneur et à l'intendant dans son mémoire du 14 mai $1726^{44}$. A partir de cette date, les congés ne sont plus remis en question. Cependant, si l'on en croit Y. Zoltvany, le système est déjà démodé ${ }^{45}$. Même durant les années où les congés sont officiellement abolis, des permis sont émis par le gouverneur et enregistrés à Montréal ${ }^{46}$. On en vient même à douter de l'abolition de 1723! De plus, de nouvelles formules d'exploitation commerciale se sont implantées et, en conséquence, plus de 25 congés ou permis sont émis chaque année.

Il est très difficile, dans l'état actuel de la recherche sur le sujet, de retracer avec précision l'évolution des différentes formes d'exploitation utilisées. Entre 1720 et 1742 s'est développée une organısation très complexe ${ }^{47}$. Le roi se réservait le commerce des postes du lac Ontario, moins profitable, sinon déficitaire, à cause de la proximité des colonies anglaises. Détroit et Michillimakinac, entrepôts de

42 Zoltvany, Vaudreuil (c1974), 164-165.

43 Lunn, Economic Development, 120.

44 Zoltvany, Vaudreuil (c1974), 207. Aussi: Filion, Maurepas, 357; Lunn, Economic Development, 121. Innis (The Fur Trade, 107) et Salone (Colonisation, 393) donnent 1728 comme date de ce rétablissement.

45 Zoltvany, Vaudreuil (c1974), 175. Innis croit, au contraire, que: "From 1728 to 1742 the licensing system was dominant" (The Fur Trade, 107).

46 E.-Z. Massicotte, "Congés et permis enregistrés à Montréal". RAPQ (19211922): $189-225$

47 Plusieurs historiens ont tenté de déterminer l'évolution des formes d'exploitation des postes. Aucun n'y a encore vraiment réussi: leur source principale, la correspondance générale, n'est pas suffisante étant donné que le gouverneur n'a pas intérêt à révéler au ministre les libertés prises, et que l'intendant n'en est pas nécessairement informé. Les archives notariales, dépouillées systématiquement, permettraient d'élucider cette question. Les renseignements sur le sujet ont été tirés de: Eccles, The Canadian Frontier, 145-147; Innis, The Fur Trade, 106-109; Lunn, Economic Development, 120-125; Salone, Colonisation, 389-393; Zoltvany, Vaudreuil (c1974), 174-175. 
la traite et établissements français permanents, étaient ouverts aux détenteurs de congés. L'accès aux autres postes se faisait, officiellement, de la même façon. Cependant, durant les dernières années de son gouvernement, Vaudreuil avait, d'après La Galissonnière ${ }^{48}$, introduit deux nouveautés dans la traite. Il avait commencé l'affermage de postes à leur commandant ou à des marchands. Il avait aussi accordé la permission à chaque commandant de poste de faire équiper deux canots pour son compte, et à chaque officier d'en faire autant avec un canot ${ }^{49}$.

Il est actuellement impossible de décrire comment cohabitaient ces différentes formes. Il est toutefois sûr qu'elles aboutirent à une augmentation rapide du nombre de canots équipés pour les pays d'en haut. Elles produisirent aussi les plaintes de marchands et des Amérindiens contre les abus des officiers commandants. Par conséquent, le roi tenta une réforme du système en 1742. Il décréta que les commandants et les officiers ne prendraient plus part au commerce. Les postes seraient affermés publiquement par mise aux enchères; Détroit et Michillimakinac, les entrepôts, feraient exception et seraient exploités par l'émission de 12 congés chacun. Le nouveau système dura jusqu'en 1749 , date où les plaintes nombreuses des fermiers amenèrent une fois encore sa révision ${ }^{50}$.

Si ces trois facteurs produisent, ensemble, une croissance du nombre de canots à destination de l'ouest ${ }^{51}$, chacun d'eux influence de façon différente l'évolution des engagements. Les guerres amérindiennes, si elles gonflent le nombre de Français dans la région des Grands lacs, n'ont par contre pas d'effet sur le nombre d'engagés. En effet, le début de la guerre contre les Renards en 1728 aurait dû grossir ce nombre: l'augmentation n'arrive que trois ans plus tard. L'expansion de la traite à l'ouest du lac Supérieur paraît par contre avoir eu un effet déterminant sur le nombre d'engagements. En effet, le début des expéditions de La Vérendrye coïncide avec une montée

48 Salone, Colonisation, 390; Zoltvany, Vaudreuil, (c 1974), 174.

49 Une preuve concrète de cette "nouveauté" se trouve dans un contrat de société pour le commerce de Chagouamigon. ANQM, Michel LePallieur, 10 sept. 1718, no 3082. Si l'on en croit Chavoy de Noyan, cette "liberté » avait pris beaucoup d'ampleur en 1738; voir Cameron Nish, Les bourgeois-gentilhommes de la Nouvelle-France, 1729-1748 ( Montréal, Fides, c1968), 79.

5) Lunn, Economic Development, 122: Innis, The Fur Trade, 107-108.

51 À partir du "Répertoire des engagements" de Massicotte, Zoltvany a estimé les nombres de canots suivants: 1720,$51 ; 1721,57 ; 1722,64 ; 1723,72 ; 1724,114$. Il n'explique pas de quelle façon il est arrivé à ces chiffres, qui ne coïncident pas avec ceux des congés. Zoltvany, Vaudreuil, (c 1974), 174-175, 184. 
très importante des engagements devant notaire: 68 en 1730, 185 en 1731. Ce ne saurait être pure coïncidence.

Les conséquences de la modification des formules d'exploitation ne sont pas aussi faciles à cerner. L'abolition des congés en 1723 et leur rétablissement en 1726 n'ont pas l'effet prévisible: le nombre d'engagements aurait dû baisser en 1724, il monte; il aurait dû au moins se maintenir en 1728. 1729 et 1730, il tombe de moitié. Quant aux autres formules d'exploitation, leur début ne peut être daté avec suffisamment de précision pour évaluer leur impact sur les engagements. Il est possible cependant qu'elles aient été mises en place au début des années 1730, plutôt que durant les dernières années du gouvernement de Vaudreuil, et que leur effet s'additionne à celui de l'expansion pour produire cette augmentation continue qui suit l'année 1731.

Ces indices suggèrent une division de l'époque des congés en deux périodes de 15 ans chacune. Elles font contraste. La première regroupe 1179 engagements, $19,8 \%$ de l'ensemble: la seconde en totalise 4168 , soit $69,9 \%$. De 1716 à 1730 , le nombre annuel d'engagements fluctue entre 46 en 1719 et 151 en 1727 ; entre 1731 et 1745, il varie de 153 en 1732 à 380 en 1738. La moyenne annuelle des quinze premières années s'élève à 78,6 engagements et elle n'est dépassée qu'à trois reprises seulement: 1724,1726 et 1727 . Si ces années exceptionnelles sont exclues, la moyenne des douze années qui restent est de 64,6 engagements. La moyenne annuelle des quinze dernières années se situe à 277,9 engagements. Seules les trois premières années en ont moins de 250 ; si elles sont exclues des calculs, la moyenne annuelle de la période grimpe à 302,8 engagements.

\section{ENGAGEMENTS ET CONGÉS: COMPARAISON QUANTITATIVE}

C'est en utilisant les congés qu'il est possible d'évaluer la représentativité des contrats retrouvés dans les archives notariales pour cette époque. En effet, l'article 4 de la Déclaration du roi du 28 avril 1716 rétablissant les congés stipule que:

Ceux qui monteront En Vertu de ses Congés dans Les postes seront tenus de Les faire Enregistrer avant de partir au greffe de La Ju'ion Royalle de montreal (...) et de Raporter (à leur retour) un Certificat du Commandant du poste ou Jls auront Eu permission Daller faire La traitte, Lequel Certifficat Jls feront 
pareillement Enregistrer aud. greffe huit jours après Leur Retour Dans La Colonie Le tout a peyne d'Estre poursuivi et pris Comme Coureurs de Bois $(\ldots)^{52}$

Les détenteurs de permissions spéciales du gouverneur devaient aussi se plier à ces exigences ${ }^{53}$. La majeure partie de ces registres ont été conservés au dépôt de Montréal des Archives nationales du Québec et ils permettent de reconstituer une série chiffrée plus ou moins complète ${ }^{54}$. Les chiffres obtenus de cette source se trouvent au tableau III.

La série des congés n'est pas complète: les renseignements manquent totalement pour sept années; ils sont incomplets pour cinq autres. La comparaison se limite donc aux années complètes. Encore une fois, le contraste est marqué entre les années 1716-1730 et les années 1731-1745. Les engagements mis en rapport avec le nombre de personnes mentionnées dans les congés donnent des pourcentages généralement inférieurs à $50 \%$ avant 1730 , presque toujours supérieurs à $50 \%$ après cette date. Les proportions totales de chacune de ces deux périodes sont révélatrices : $38,7 \%$ et $62,6 \%$.

52 ANQM, Registre des audiences, 8: f. 1250. Les certificats de retour n'ont pu être localisés. Ils auraient pourtant été très précieux pour effectuer d'autres vérifications et, peutêtre, mieux connaître ceux qui ne revenaient pas.

53 Ibid.: ff. 1245v-1247, art. 2, 5, 7, 8.

54 Des listes de congés ont déjà été publiées: "Les congés de traite accordés en 1717 ", BRH, XXIX, 9 ( sept. 1923): 271-274; E.-Z. Massicotte, "Congés et permis déposés ou enregistrés à Montréal sous le Régime français ", RAPQ(1921-1922): 189-255; E.-Z. Måssicotte, "À propos de congés", BR H, XXXII, 5 (mai 1926): 296-300 (complète le précédent): "Les congés de traite sous le Régime français au Canada (Congés de traite conservés aux Archives de la province de Québec)", RAPQ (1922-1923): 191-265. La liste de Massicotte est tirée de deux registres de congés (reliés en un seul volume): ANQM, Enregistrement de congés. ordonnances Et Arrêts Commencé le 31 mars 172I(1721-20 août 1726), et un second registre sans page-titre couvrant la période du 3 juillet 1728 au 13 juin 1730. Les ANQM conservent d'autres registres de congés, que Massicotte n’a pas trouvés pour la bonne raison qu'ils ont été reliés avec les Registres des audiences: congés de 1717 et 1718, vol. 8(1714-1719); congés de 1719 et 1720, vol. 9(1719-1722): «Livre des Enregistrements des Congés du 31e mai 1731 (au 8 juillet 1732)", vol. 12 (1730-1732); "( Registre des congés, du 21 mai 1733 au ler juin 1734 )", vol. 14 (1733-1734); "Registre Pour les Congés Commencé Le 2e juin 1734 (au 12 juillet 1735)", vol. 15 (1734-1736); "1744. Congés", vol. 23 (1744-1746). Claude Richard nous a fourni ces indications. Les congés des années 1739 à 1745 ont été inventoriés dans RAPQ(1922-1923). Même les données qui ont été considérées comme complètes risquent de ne pas l'être. Pour s'en assurer, il faudrait dépouiller tous les registres conservés aux ANQM. Il semble, en effet, que le greffier n'ait pas toujours enregistré le même type de document au même registre. Raymond Dumais, des ANQM, a signalé 12 congés pour 1732 ( 18 canots, 84 hommes) enregistrés parmi les insinuations: ANQM, Registre des Insinuations: Contrats de mariage, et autres Actes..., 15 juillet-19 août 1732: Livre des Insinuations Et contrats de mariage, des donations et Congés.... 18 août-2 oct 1732 . 


\begin{tabular}{|c|c|c|c|c|}
\hline \multicolumn{5}{|c|}{$\begin{array}{c}\text { TABLEAU III } \\
\text { Engagements et congés, 1716-1745 } \\
\text { Statistiques annuelles }\end{array}$} \\
\hline \multirow[b]{2}{*}{ Années } & Notaires & \multicolumn{2}{|c|}{ Congés } & \multirow{2}{*}{$\begin{array}{l}\text { Proportion } \\
\text { Eng. / personnes } \\
\%\end{array}$} \\
\hline & $\begin{array}{l}\text { Engagés } \\
\text { Nombre }\end{array}$ & $\begin{array}{l}\text { Personnes } \\
\text { nombre }\end{array}$ & $\begin{array}{l}\text { Canots } \\
\text { nombre }\end{array}$ & \\
\hline 1716 & 58 & - & - & - \\
\hline 1717 & 66 & $(12)^{a}$ & ( 4) & $(550,0)$ \\
\hline 1718 & 56 & $90 \mathrm{~b}$ & 23 & 62,2 \\
\hline 1719 & 46 & $83 \mathrm{c}$ & 21 & 55,4 \\
\hline 1720 & 47 & $118^{\mathrm{c}}$ & 30 & 39,8 \\
\hline 1721 & 75 & $266^{c}$ & 57 & 28,2 \\
\hline 1722 & 67 & 231 & 58 & 29,0 \\
\hline 1723 & 78 & 178 & 40 & 43,8 \\
\hline 1724 & 112 & 138 & 30 & 81,2 \\
\hline 1725 & 73 & 238 & 52 & 30,7 \\
\hline 1726 & 141 & $(141)^{\mathrm{d}}$ & $(31)$ & $(100,0)$ \\
\hline 1727 & 151 & - & - & \\
\hline 1728 & 73 & $(187)^{\mathrm{e}}$ & (39) & $(39,0)$ \\
\hline 1729 & 68 & 264 & 54 & 25,6 \\
\hline 1730 & 68 & $(136)^{f}$ & (30) & $(50,0)$ \\
\hline 1731 & 185 & $(242) \mathrm{g}$ & (49) & $(76,4)$ \\
\hline 1732 & 153 & 344 & 71 & 44,5 \\
\hline 1733 & 196 & 422 & 80 & 46,4 \\
\hline 1734 & 296 & 438 & 79 & 67,6 \\
\hline 1735 & 256 & 388 & 69 & 66,0 \\
\hline 1736 & 299 & - & - & \\
\hline 1737 & 286 & - & - & \\
\hline 1738 & 380 & - & - & \\
\hline 1739 & 271 & 393 & 68 & 69,0 \\
\hline 1740 & 285 & 318 & 54 & 89,6 \\
\hline 1741 & 351 & - & - & \\
\hline 1742 & 319 & - & - & \\
\hline 1743 & 316 & 469 & 78 & 67,4 \\
\hline 1744 & 289 & 551 & 91 & 52,4 \\
\hline 1745 & 286 & 430 & 70 & 66,5 \\
\hline \multicolumn{5}{|c|}{ a. Cette année est manifestement incomplète. } \\
\hline \multicolumn{5}{|c|}{$\begin{array}{l}\text { b. Les données de } 1718 \text { proviennent de } \mathrm{BRH} \text {, vol. XXIX. Celles du registre indiquant } 4 \\
\text { canots et } 15 \text { hommes sont incomplètes. }\end{array}$} \\
\hline \multicolumn{5}{|c|}{$\begin{array}{l}\text { c. Cinq canots de } 4 \text { hommes chacun, partis en juin } 1719 \text {, n'ont été enregistrés que les } 25 \text { mai } \\
30 \text { mai, } 4 \text { juin et } 6 \text { août } 1720 \text {, soit longtemps après leur départ. De même, un canot pour } \\
\text { les Ouiatanons, enregistré en avril 1721, partit en septembre } 1719 \text {. }\end{array}$} \\
\hline \multicolumn{5}{|c|}{$\begin{array}{l}\text { d. Il manque peut-être des congés pour } 1726 \text { : la dernière permission enregistrée l'est le } \\
20 \text { août. }\end{array}$} \\
\hline \multicolumn{5}{|c|}{ e. Les enregistrements pour 1728 ne commencent que le 3 juillet. } \\
\hline \multicolumn{5}{|c|}{ f. Le dernier enregistrement pour 1730 a été fait le 13 juin. } \\
\hline Le pren & gistremen & 1 date du 3 & & \\
\hline
\end{tabular}




\section{GRAPHIQUE II}

Engagements et congés

(1716-1745)

\# Nombre de personnes selon les congés

- Nombre d'engagements

-- Moyenne annuelle des engagements

(A) 1716-1730; (B) 1731-1745

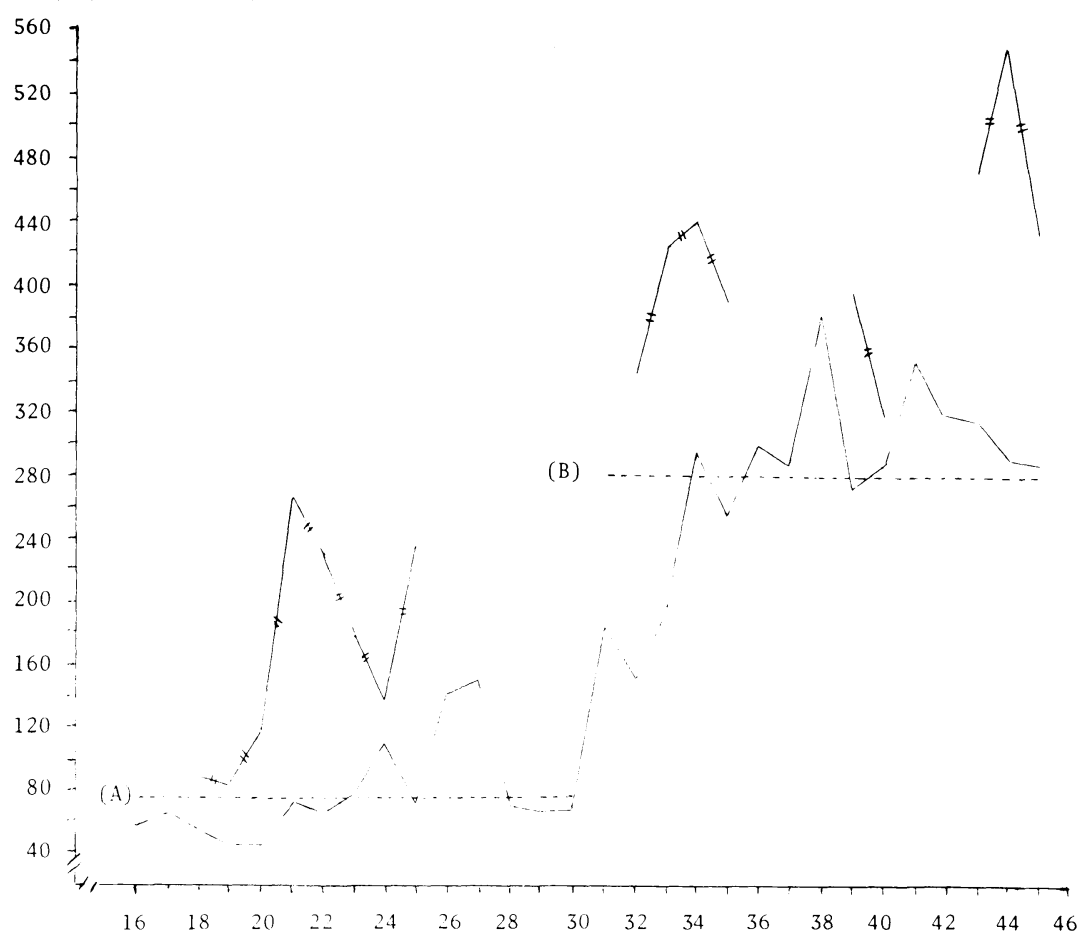

TABLEAU IV

Engagements et congés

(années complètes)

$\begin{array}{lccc}\text { Années } & \text { Engagements } & \text { Congés } & \\ 1716-1730 & 622 & 1606 & 38,7 \\ 1731-1745 & 2348 & 3753 & 62.6 \\ \text { TOTAL } & 2970 & 5359 & 55.4\end{array}$


Le nombre de personnes inscrit au congé n'est toutefois pas égal au nombre d'employés. Il inclut souvent partenaires ou employeurs, et la distinction entre les deux n'est pas toujours claire. Toute risquée que soit l'opération, cette distinction a été faite pour certaines années, afin d'indiquer la tendance. En 1718, selon les congés, 90 hommes quittaient Montréal pour l'ouest à bord de 23 canots; 34 hommes étaient fort probablement employeurs ou partenaires et 56, des employés. Comme 56 engagements ont été retrouvés, tous les employés auraient signé un contrat cette année-là. La situation est tout à fait différente en 1729. Des 264 hommes mentionnés dans les congés, 29 au maximum peuvent être des partenaires ou des employeurs. Les notaires ont recueilli 68 engagements, soit $28,9 \%$ des 235 engagés mentionnés dans les congés. En 1739 , seulement 31 des 393 personnes indiquées dans les congés pourraient être employeurs ou partenaires: $74,9 \%$ des contrats auraient été retrouvés. Enfin, en 1745, 5 hommes seulement mentionnés dans les congés peuvent être considérés comme des employeurs.

Si ces renseignements, partiels il va sans dire, sont ajoutés à ceux concernant la période précédente, la tendance vers la concentration de la traite entre les mains d'un petit groupe de personnes se confirme. Comme l'écrit Cameron Nish, «un groupe restreint d'officiers et de marchands dominaient la traite des fourrures en Nouvelle-France pendant les années 1729 à 1748 " 55 . De sorte que la proportion d'employés en 1745 est très élevée comparée à ce qu'elle était en 1718. Toutefois, il est impossible d'expliquer au moyen des renseignements recueillis pourquoi il y a, entre 1730 et 1731 , une coupure au niveau des contrats notariés. Celle-ci ne semble pas exister dans les congés et, d'après les données de 1729 , la concentration est alors déjà très poussée.

\section{ENGAGEMENTS ET CONGÉS (1743-1745): JUMELAGE DES DONNÉES}

Il y a un autre indicateur de la valeur de ces sources: le jumelage des noms de personnes rencontrées dans l'une ou l'autre. À part quelques-uns, les congés enregistrés en 1743, 1744 et 1745 sont suivis du "rolle" de l'équipage des canots. L'opération consiste donc à retrouver dans la liste des engagés les noms apparaissant dans ces «rolles». L'opération est pleine d'embûches, comme le savent bien

55 Cameron Nish, Les Bourgeois-gentilshommes, 98. Tout le chapitre V. "La Bourgeoisie et les Pays d'en haut", est consacré à l'étude de cette question. 
les adeptes de la démographie historique et de la reconstitution de familles ${ }^{56}$. Les congés et les engagements pour ces trois années contiennent au total 2317 noms. Théoriquement, ces noms peuvent représenter autant de personnes différentes. De plus, la même personne peut être inscrite sous son nom de famille ou sous son surnom. Á cause du nombre et de cette difficulté, le jumelage est sommaire.

La règle de base est simple: un nom inscrit au bas d'un congé et se retrouvant sur un contrat d'engagement a été considéré comme désignant une seule personne lorsque le nom de famille et / ou le surnom et le prénom de même que le lieu de résidence, lorsqu'il était indiqué, étaient identiques. Très peu de libertés ont été prises; il s'agit de cas où l'orthographe du nom était différent mais la consonance demeurait la même. Dans plusieurs cas, où le nom de famille seul était inscrit au congé, le jumelage n'a pas été fait même si l'originalité du nom (e.g. Labombarde), le lieu de résidence, l'employeur et la destination inscrite concordaient exactement. Ce conservatisme visait à éviter de confondre deux frères. Le jumelage est donc minimal. Les résultats de l'opération sont inscrits au tableau $\mathrm{V}$.

\section{TABLEAU V}

\section{Engagements et congés - jumelage des données}

\begin{tabular}{|c|c|c|c|c|c|c|c|c|}
\hline \multirow[b]{3}{*}{ Année } & \multicolumn{3}{|c|}{ Noms retrouvés } & \multicolumn{5}{|c|}{ Noms jumelés } \\
\hline & $\mathrm{Co}$ & gés & Contrats & & & & & \\
\hline & $\begin{array}{l}\text { inscrits } \\
\text { (1) }\end{array}$ & $\begin{array}{c}\text { complets } \\
\text { (2) }\end{array}$ & (3) & nombre & $\%$ de 1 & (\%) de 2 & $\because$ de 3 & Total ${ }^{a}$ \\
\hline 1743 & 440 & 370 & 317 & 166 & 37,7 & 44,9 & 52,4 & 591 \\
\hline 1744 & 534 & 487 & 288 & 187 & 35,0 & 38,4 & 64.9 & 635 \\
\hline 1745 & 452 & 373 & 286 & 138 & 30,5 & 37,0 & 48,2 & 600 \\
\hline TOTAL & 1426 & 1230 & 891 & 491 & 34,4 & 39,9 & 55.1 & 1826 \\
\hline
\end{tabular}

a. Ce total est formé des noms inscrits aux congés (1) plus ceux des contrats (3) moins les noms jumelés.

L'opération jumelage donne des résultats surprenants : $34,4 \%$ des noms inscrits au bas des congés se retrouvent dans les contrats d'engagement et $55,1 \%$ des noms recueillis dans ces derniers sont

56 Le dernier article paru en langue française sur le sujet: Gérard Bouchard et Patrick Brard, "Le programme de reconstitution automatique des familles saguenayennes: données de base et résultats provisoires", Histoire sociale / Social History, XII, 23 (mai-May 1979): 170-185. 
également au bas des congés. Les deux séries ne concordent donc pas, et de loin. Ce qui permet quelques hypothèses. D'abord, il est possible que le nombre total des personnes en partance pour l'ouest en une année donnée soit plus élevé que ce qu'indiquent les congés. Si c'était le cas, 1826 personnes auraient quitté Montréal pour l'ouest durant ces trois années et seulement 891 , soit $48,8 \%$ auraient passé contrat devant notaire. C'est $13,1 \%$ plus bas que ce que révélaient les statistiques annuelles ( tableau III). Il est ensuite possible que certains employés aient décidé de ne pas faire le voyage qu'ils s'étaient engagés de faire par contrat. Ils auraient omis de faire annuler leur contrat ou se seraient fait remplacer. Si cette seconde hypothèse se vérifiait, la valeur et la représentativité des contrats passés devant notaire seraient considérablement réduites.

Il faut cependant tenir compte d'un autre facteur: la procédure suivie dans l'un et l'autre cas. Elle donne plus de poids à la liste d'engagements. En effet, les contrats d'engagement ont été passés devant notaire en présence de deux témoins. Sauf quelques rares cas, l'engagé est présent. Une fois signé, le contrat a atteint sa forme définitive. Toutefois, une certaine distorsion des données peut se produire lors de la cueillette des données. Par contre, le contrat devant notaire n'est pas obligatoire pour lier les parties puisqu'un contrat sous seing privé et même une entente verbale peuvent remplir à peu près le même rôle qu'un contrat devant notaire.

L'enregistrement des congés comporte plusieurs étapes et chacune augmente le risque de distorsion et d'erreurs. Il s'agit d'une déclaration du détenteur du congé au greffier de la juridiction royale de Montréal. L'employeur pourrait bien oublier certains noms, comme il le fait pour certains prénoms. Si le greffier n'inscrit pas directement au registre la liste fournie par l'employeur ${ }^{57}$, des erreurs peuvent bien se glisser lors de la transcription. Enfin, comme pour les engagements, la cueillette des données n'est pas sans danger. Ces trois étapes, déclaration de l'employeur et transcriptions par le greffier puis par le chercheur, multiplient les risques d'erreurs, risques moindres lors de la seule étape du dépouillement des engagements par le chercheur.

L'analyse de ces divers facteurs aboutit à la formulation de propositions relatives à la valeur des sources pour 1743, 1744 et 1745. Comme le détenteur du congé est tenu par la Déclaration de rétablissement de faire enregistrer son congé ainsi que le nombre et

57 C'est la procédure suivie pour les audiences de la juridiction royale. 
le nom des hommes composant l'équipage, cette série devrait être relativement fiable quant à la quantité d’hommes employés. Cette proposition est aussi valable pour les années précédentes. Pour ce qui est de l'identité des hommes, elle est absolument sûre lorsque leur nom se retrouve dans les engagements et les congés; elle est relativement sûre lorsqu'il se retrouve dans un contrat d'engagement, et moins sûre lorsqu'il se trouve au bas d'un congé.

La «production» de fourrures, si elle était connue, pourrait être utilisée pour évaluer la documentation tirée des archives notariales. Seule est connue actuellement la valeur des fourrures importées à La Rochelle ${ }^{58}$. Ces importations, même diminuées plus ou moins arbitrairement des fourrures provenant de colonies autres que la Nouvelle-France, ne peuvent être considérées comme égales à la production. Elles ne tiennent pas compte des pertes en mer ni des entrées dans les autres ports de France; elles excluent la contrebande vers les colonies anglaises. Comme leur valeur, plutôt que leur volume, est inscrite, elles peuvent fluctuer selon les prix utilisés pour évaluer les fourrures à l'entrée: prix de Nouvelle-France, prix de La Rochelle, prix déclaré par le capitaine.

Même en supposant que ces facteurs n'aient qu'un effet mineur sur les statistiques des importations, elles ne seraient pas encore comparables aux engagements devant notaire, ou au personnel de la traite d'après les congés. Pour pouvoir faire la comparaison, il faudrait plutôt connaître le nombre d'engagés revenant des pays d'en haut, avec les fourrures. Ceux qui s'engagent ne reviennent pas tous la même année. À cause de toutes ces restrictions, il est préférable de ne pas utiliser cette documentation. Les livres de compte des marchands n'ont pas été utilisés pour cette recherche. Ceux qui ont été préservés sont trop peu nombreux pour être vraiment utiles. De plus, ils ne se comparent pas au livre des engagés de la collection Ermatinger, dont Ouellet s'est servi ${ }^{59}$.

\section{CONCLUSION}

Les contrats d'engagement pour la traite ne comprennent pas tous les engagements. Leur valeur documentaire et leur représenta-

58 Émile Garnault, Les Rochelois et le Canada, 14-16, cité dans Salone, Colonisation, 397-398: Lunn, Economic Development, 464-465. Cette dernière a déduit les fourrures provenant d'autres colonies, ce que n’a pas fait Salone, ni Filion (Maurepas, 331 ).

59 L. Dechêne a étudié à fond ceux d'Alexis Monière pour les années 1715 à 1724. Dans son tableau, elle ne fait toutefois pas la distinction entre voyageurs et engagés ( $\mathrm{Habi}$ tants et marchands, 184). F. Ouellet, "Dualité économique», 268-269. 
tivité varient selon les périodes. Tant que dure la Compagnie de la colonie, on peut considérer que la très grande majorité des engagés, sinon tous, passent contrat devant notaire. Entre 1706 et 1716, les conditions de la traite favorisent l'association plutôt que l'engagement; par conséquent, les engagés sont peu nombreux et le petit nombre de contrats retrouvés est représentatif d'une situation réelle.

Avec le rétablissement des congés, les générosités des gouverneurs et l'affermage des postes, se confirme la tendance à la concentration du commerce des fourrures entre les mains d'un groupe restreint d'individus. À partir de 1716, la proportion d'engagés indiquée par les congés augmente d'année en année. La documentation devient alors plus difficile à évaluer. De 1716 à 1730 , la liste des contrats est de moins en moins représentative de l'ensemble de la situation, comme l'indique la comparaison des contrats aux congés. La situation s'améliore nettement à partir de 1731 , alors que les engagements représentent $62,6 \%$ des personnes retrouvées dans les congés. Le jumelage des données pour les années 1743 et 1745 amène toutefois à conclure que ni les congés ni les contrats d'engagement ne sont entièrement fiables.

Les comparaisons faites entre les contrats d'engagement et d'autres sources documentaires relatives au personnel de la traite font ressortir que les contrats sont suffisamment représentatifs pour permettre l'étude des conditions d'engagement et, à l'instar de L. Dechêne, pour peindre un tableau valable. Cependant, si l'on veut pousser l'étude plus loin et reconstituer les allées et venues de ces engagés ou d'une partie d'entre eux, il faudra être très prudent. En effet, les actes notariés, particulièrement après 1716, ne peuvent pas "être considérés comme de véritables registres d'enregistrement des allées et venues dans l'Ouest». 